\title{
Classification of radical hysterectomy adopted by the Gynecological Cancer Group of the European Organization for Research and Treatment of Cancer $^{1}$
}

\author{
F. MOTA*, I. VERGOTE†, J.B. TRIMBOS‡, F. AMANT†, N. SIDDIQUI§, A. DEL RIO\|, \\ R. VERHEIJEN9 \& P. ZOLA** \\ *Department of Gynecology, University Hospital of Coimbra, Coimbra, Portugal; †Division of Gynecologic Oncology, \\ University Hospitals Leuven, Leuven, Belgium; ¥Department of Gynecology, Leiden University Medical Center, \\ Leiden, The Netherlands; §Department of Gynecologic Oncology, North Glasgow University Hospital, Glasgow, UK; \\ //Departamento de Obstetricia y Ginecologia, Hospital General de Asturias, Oviedo, Spain; qDepartment of Obstetrics \\ and Gynecology, Vrije Universiteit Medical Center, The Netherlands; and **Department of Gynecology and Obstetrics, \\ University of Turin, Turin, Italy
}

\begin{abstract}
Mota F, Vergote I, Trimbos JB, Amant F, Siddiqui N, Del Rio A, Verheijen R, Zola P. Classification of radical hysterectomy adopted by the Gynecological Cancer Group of the European Organization for Research and Treatment of Cancer. Int J Gynecol Cancer 2008;18:1136-1138.

The Piver classification of radical hysterectomy for the treatment of cervical cancer is outdated and misused. The Surgery Committee of the Gynecological Cancer Group of the European Organization for Research and Treatment of Cancer (EORTC) produced, approved, and adopted a revised classification. It is hoped that at least within the EORTC participating centers, a standardization of procedures is achieved. The clinical indications of the new classification are discussed.
\end{abstract}

KEYWORDS: cervix cancer, classification of radical hysterectomy, EORTC.

There are several surgical procedures that can be used in treating women with early-stage cervical cancer. The majority of the gynecological surgeons have adopted the five classes of the Piver-Rutledge-Smith classification of radical hysterectomy published in $1974^{(1)}$. However, several criticisms have been made to this classification, and modifications have been proposed, which resulted in its misuse by surgeons and centers around the world. This translates in striking differences in the radicality of the procedures, thereby not allowing precise comparisons and accurate future documentation of survival (curative effect of the surgery), as well

Address correspondence and reprint requests to: Fernando Mota, $\mathrm{MD}, \mathrm{PhD}$, Department of Gynecology, University Hospital of Coimbra, 3000 Coimbra, Portugal. Email: fmota@huc.min-saude.pt

F. Mota is the Chairman of the Surgery Committee of the Gynecological Cancer Group of the European Organization for Research and Treatment of Cancer.

${ }^{1}$ Classification approved by the Surgery Committee and adopted by the Gynecological Cancer Group of the European Organization for Research and Treatment of Cancer, Brussels, March 2007.

doi:10.1111/j.1525-1438.2007.01138.x as treatment-related (surgical) morbidity. Hence, the Surgery Committee of the Gynecological Cancer Group (GCG) of the European Organization for Research and Treatment of Cancer (EORTC) produced, approved, and adopted a revised version of the original Piver classification of radical hysterectomy. The goal of this new classification is to clarify several surgical details of the widely used but outdated Piver classification and make it more practical, clinically more relevant, and particularly to allow standardization of procedures among oncology departments around Europe that participate in clinical trials within the EORTC.

\section{Classification of radical hysterectomy adopted by the GCG of the EORTC}

1. Simple hysterectomy (type I).

2. Modified radical hysterectomy (type II): The uterus, paracervical tissues, and upper vagina (1$2 \mathrm{~cm}$ ) are removed after dissection of the ureters to the point of their entry to the bladder. The uterine arteries are ligated, and the medial half of the 
parametria (there was no consensus in this concept) and proximal uterosacral ligaments are resected.

3. Radical hysterectomy (type III): En bloc removal of the uterus with the upper third of the vagina along with the paravaginal and paracervical tissues. The uterine vessels are ligated at their origin, and the entire width of the parametria is resected bilaterally. Removal of as much of the uterosacral ligaments as possible.

4. Extended radical hysterectomy (type IV): Differ from the type III procedure-three fourths of the vagina and paravaginal tissue are excised.

5. Partial exenteration (type V): The terminal ureter or a segment of the bladder or rectum is removed along with the uterus and parametria (supralevatorial).

A) Types II-V hysterectomies are completed with a systematic bilateral pelvic lymphadenectomy, half the way along the common iliac artery down to the femoral ring, including the presacral, both external, internal, and interiliac and the obturator nodes (at least to the level of the obturator nerve). B) Removal of the tubes and ovaries is not part of radical hysterectomy per se.

\section{Discussion}

The GCG of the EORTC classification of radical hysterectomy is a revised and updated version of the original Piver classification. Therefore, concerns over its validation are not an issue since the Piver classification has been validated for several decades of clinical use and a few randomized trials.

Both classifications (original Piver and GCG of the EORTC-adopted classification) include a type I hysterectomy, which is not, in fact, a radical hysterectomy. However, it is included in the revised classification because it is accepted as a possible procedure for the treatment of stage IA1 cervical cancer without lymphvascular space invasion ${ }^{(2)}$. Contrary to the Piver terminology, which refers to the type I hysterectomy as an "extrafascial" hysterectomy, the GCG of the EORTC-adopted classification uses the broader term "simple" hysterectomy since vaginal and laparoscopic approaches have been used increasingly.

There is no agreement within the surgical oncology community on the definition of parametrium and its anatomical boundaries. This is reflected on the terminology used in the updated classification of types II and III radical hysterectomies. Furthermore, the type
II hysterectomy adopted by the GCG of the EORTC does not define the level at which the uterine vessels should be ligated since this has neither oncologic significance nor impact on morbidity.

Some surgeons subdivide type II radical hysterectomy into IIA - the parametria are resected at the level where the uterine artery crosses the ureter-and IIB - the medial half of the parametria are resected. However, this is controversial and lacks clinical relevance. In general, type II radical hysterectomy is indicated for the treatment of stage IA1 with extensive lymphvascular space invasion, stage IA2, and small stage IB1 ( $<1 \mathrm{~cm}$ stromal invasion) cervical cancers ${ }^{(2)}$. For these microinvasive and very small early-stage cervical cancers, there is no need to remove the upper one third of the vagina, as described by Piver et al. ${ }^{(1)}$. The removal of the upper 1-2 cm of the vagina has been documented to be adequate ${ }^{(3)}$.

Type III radical hysterectomy is indicated for stages IB1 and IIA (invasion of the upper vagina) cervical cancers $^{(3,4)}$. Although controversial, a few clinicians also advocate radical hysterectomy for stage IIB cervical cancers.

The GCG of the EORTC definition of type IV radical hysterectomy differs significantly from the corresponding Piver classification. The original classification establishes that "the ureter is completely dissected from the pubo-vesicle ligament" and "the superior vesicle artery is sacrificed." In order to perform such an extended type IV hysterectomy, the ureter needs to be completely dissected and isolated. Also, the superior vesicle artery may not necessarily need to be sacrificed. Moreover, this sacrifice adds nothing to the radicality of the procedure. Type IV radical hysterectomy may be indicated for the treatment of large stage IIA cervical tumors, when adequate surgical margins are judged to be attainable.

Partial exenteration (type V), either anterior or posterior, is rarely used bearing in mind the high morbidity of the procedure and because new protocols with concurrent chemoradiotherapy seem to be a superior alternative. The GCG of the EORTC classification takes into account the possibility of performing surgery either on the bladder or on the colon. Medically fit patients with central and isolated pelvic recurrences or persistent disease after primary (chemo)radiotherapy may be candidates to type $\mathrm{V}$ radical hysterectomy ${ }^{(5)}$ or other forms of ultraradical surgical techniques.

The original Piver classification ignored the extent of pelvic lymph node dissection, which is considered in the GCG of the EORTC classification. It is unnecessary to comment on the paramount importance of 


\section{F. Mota et al.}

lymph node dissection in early-stage cervical cancer in terms of both prognosis (nodal status) and survival (therapeutic impact). It is generally acknowledged, as within the GCG of the EORTC, that 12 pelvic lymph nodes are the minimal sampling acceptable. Some surgeons start lymphadenectomy at the level of the bifurcation of the aorta, while many others start half the way along the common iliac artery, as recommended by the GCG Surgery Committee of the EORTC. Any palpable bulky para-aortic node should also be resected, given that radiation therapy cannot sterilize metastatic nodes larger than $2 \mathrm{~cm}$ in diameter ${ }^{(6)}$.

Since removal of the tubes and ovaries is not part of the radical hysterectomy per se, it is not described. Nevertheless, it should be emphasized that for women younger than 40-45 years, the ovaries usually are not removed and should be transposed into the paracolic gutters out of the pelvis. The incidence of ovarian metastases in early-stage cervical cancer is minimal, even in the presence of pelvic node metastases $^{(7)}$.

Future improvements of the revised classification of radical hysterectomy adopted by the GCG of the
EORTC should take into account the precise definition (embryologic and anatomical) of the parametrium, as well as consider the nerve-sparing techniques and fertility-preserving surgery.

\section{References}

1 Piver MS, Rutledge F, Smith JP. Five classes of extended hysterectomy for women with cervical cancer. Obstet Gynecol 1974;44:265-72.

2 Mota F. Microinvasive squamous carcinoma of the cervix: treatment modalities. Acta Obstet Gynecol Scand 2003;83:1-5.

3 Landoni F, Maneo A, Cormio G et al. Class II versus class III radical hysterectomy in stage IB-IIA cervical cancer: a prospective randomized study. Gynecol Oncol 2001;80:3-12.

4 Landoni F, Maneo A, Colombo A et al. Randomized study of radical surgery versus radiotherapy for stage IB-IIA cervical cancer. Lancet 1997;350:535-40.

5 Rutledge S, Carey MS, Pritchard $\mathrm{H}$ et al. Conservative surgery for recurrent or persistent carcinoma of the cervix following irradiation: is exenteration always necessary? Gynecol Oncol 1994;52:353-9.

6 Vigliotti AP, Wen BC, Hussey DH et al. Extended field irradiation for carcinoma of the uterine cervix with positive periaortic nodes. Int $J$ Radiot Oncol Biol Phys 1992;23:501-9.

7 Landoni F, Zanagnolo V, Lovato-Diaz L et al. Ovarian metastases in early-stage cervical cancer (IA2-IIA): a multicenter retrospective study of 1965 patients (a Cooperative Task Force study). Int J Gynecol Cancer 2007;17:623-8.

Accepted for publication August 20, 2007 